\title{
A Unified RGPS-Based Approach Supporting Service-Oriented Process Customization
}

Jian Wang, Zaiwen Feng, Jia Zhang, Patrick C. K. Hung, Keqing He, and Liang-Jie Zhang

\section{Introduction}

Cloud computing is defined as a model for enabling ubiquitous, convenient, ondemand network access to a shared pool of configurable computing resources [15]. This has created significant improvements in terms of software development. Software as a Service (SaaS) is a core objective of cloud computing, aiming to provide utility-oriented software delivery and provisioning. Increasing amounts of software have been published as reusable Web APIs or Web services (e.g., over 28,000 services and 7,000 APIs have been published in Seekda! ${ }^{1}$ and ProgrammableWeb ${ }^{2}$ by September 2012, respectively), allowing users to quickly compose multiple services into a new value-added process, also known as a composite service or a mashup. However, various users may have personalized requirements, thus preferring to

Jian Wang

State Key Laboratory of Software Engineering, School of Computer, Wuhan University, China, e-mail: jianwang@whu.edu.cn

Zaiwen Feng

State Key Laboratory of Software Engineering, School of Computer, Wuhan University, China, e-mail: fengzaiwen@whu.edu.cn

Jia Zhang

Carnegie Mellon University - Silicon Valley, USA, e-mail: jia.zhang@sv.cmu.edu

Patrick C. K. Hung

University of Ontario Institute of Technology, Canada, e-mail: patrick.hung @uoit.ca

Keqing $\mathrm{He}$

State Key Laboratory of Software Engineering, School of Computer, Wuhan University, China, e-mail: hekeqing@whu.edu.cn

Liang-Jie zhang

Kingdee International Software Group Co. Ltd., China, e-mail: zhanglj@ieee.org

${ }^{1}$ http://webservices.seekda.com/

2 http://www.programmableweb.com 
compose the same set of software services in different ways. Meanwhile, the large amount of available services makes it difficult for end-users to select suitable services. Therefore, there is a need to provide a unified way for users to configure and compose software services according to their personalized requirements.

WS-BPEL [18] is the most widely adopted standard for building business processes using Web services. Many works have extended WS-BPEL to achieve more flexible and customizable process modeling. For example, Mietzner et al. transform variability descriptors into a WS-BPEL process model to guide a customer through the customization of an SaaS application [16]; Lazovik et al. define a formal model to guide obtaining customer-specific process instantiations by a series of customization steps over reference processes [11]; Van der Aalst et al. investigate the workflow variants within the context of configurable workflow models [21]. However, these works do not consider service-oriented process customization in terms of various end user requirements. The works [3, 9] establish relationships between process models and requirements models such as KAOS and $i^{*}$, followed by generating, validating and configuring process models. Compared with these works, our work will focus on customizing service-based processes in a holistic way by connecting the requirements models (role $\&$ goal models), process models, and services.

The RGPS (Role-Goal-Process-Service) meta-model framework [5, 22] has been proposed for describing personalized requirements involving domain-related services, toward an ultimate goal of enabling on-demand service provisioning. RGPS is an integral part of the ISO Meta-model Framework for Interoperability $\left(\mathrm{MFI}^{3}\right)$. This chapter presents an approach that leverages RGPS to help users dynamically configure a personalized service-based mashup. RGPS-based domain modeling is used to create domain models that will be reused in the subsequent process customization. Faced with a user' specified requirements, a hierarchical goal tree can be generated for users to refine their specific business goals. According to mappings between goals and variation points in business process templates, a personalized business process model will be created. Afterwards, each task in the process model may be realized by an external service.

Based on the proposed approach, corresponding visualization prototypes including a domain modeling tool and a service supermarket are introduced to assist users in such a RGPS-based service-oriented process configuration. This chapter also describes a case study of customizing a mashup over an established service supermarket consisting of a set of Web services, including a contact service, Delivery-100 API, Check RFID service, Baidu map API, and Yahoo! weather API.

The remainder of the chapter is organized as follows. Section 2 introduces our motivation followed by an overview of our approach. Section 3 gives an introduction of the RGPS meta-model framework. The RGPS-based service-oriented process customization approach is presented in section 4. Section 5 shows the visualization prototypes that support the proposed approach. A case study is given in section 6 . Section 7 concludes the chapter.

\footnotetext{
${ }^{3}$ http://metadata-stds.org/19763/index.html
} 


\section{Motivation and Overall Approach}

In this section, a motivating example will be given, followed by an overview of our approach.

\subsection{A motivating example}

The following scenario in the Customer Relationship Management(CRM) domain shows a motivating example of our work. Suppose a salesman in a company plans to deliver goods to his customer. First, he needs to acquire the recipient's shipping information such as address, phone, and postcode. He then will select a shipping company to send these goods and generate a shipping order. After that, he will use a payment service to pay for the order. Finally, after the goods is sent to the shipping company, he can track the shipping status of his goods. Usually, the user can query the order to get the status of the goods in text. However, many salesmen may have additional requirements in tracking the order. For example, some people may want to view the current location of the goods on a map during the shipping process, while some people may hope to know the current weather information of the cities in the shipping route, which can be used to judge whether their goods can arrive at the destination in time. In addition, if the goods are expensive and brittle, some salesmen may hope to check the integrity of these goods during the shipping process.

From this simple scenario, we can see that different users' requirements may be different towards the same goal of "delivering goods to customers." Moreover, initial requirements may be incomplete, and additional requirements may be added or refined during the process of requirements analysis. So it is almost impossible to find a single service that can satisfy the salesman's requirements. Automatic service composition will also be hard to work due to the variability and incompleteness of users' personalized requirements. Therefore, there is a need to provide a unified way for users to configure and compose services according to users' requirements.

\subsection{Overall Approach}

Towards the above-mentioned problem, we propose a RGPS-based service-oriented process customization solution. The RGPS meta-model framework describes requirements and service-based solution in a holistic way using four key elements: Role, Goal, Process, and Service, which provides a guideline for our approach. Our approach is a domain-oriented solution, since domain knowledge can be used to supplement the incomplete requirements and serve as a communication bridge between users and developers to avoid ambiguity. Moreover, many researchers have shown that it is more suitable to reuse software assets in a specific domain [17], and 
Fig. 1 Our overall approach

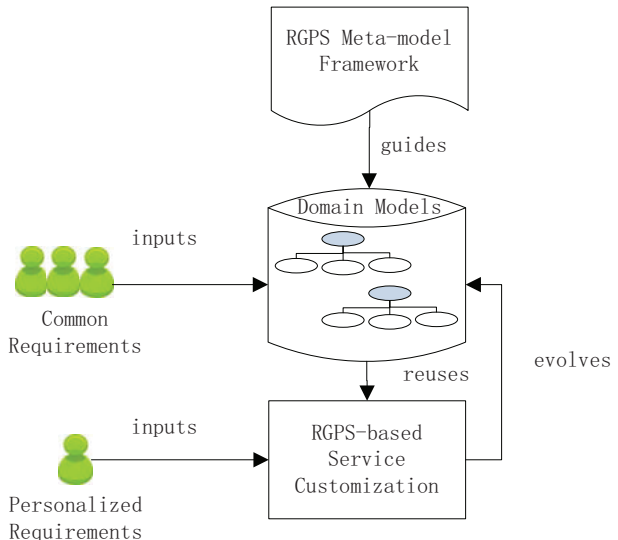

services as important software assets are no exception. Therefore, domain modeling will play an important role during process customization.

As shown in Fig. 1, the RGPS meta-model framework will provide a guideline for our approach. Based on users' common requirements in a specific domain, domain models can be created by identifying the commonality and variability of domain knowledge. Then, faced with a user' personalized requirements, RGPS-based service-oriented process customization process can be conducted to provide solutions based on domain models. The result of customization process will in turn contribute to the evolution of domain models. Note that in this chapter, we mainly describe how to customize service-oriented processes, and did not introduce the evolution of domain models. We will explain our approach using the motivating example in Sect. 2.1 throughout the chapter.

\section{The RGPS Meta-model Framework}

To support service-oriented software development, we have proposed a meta-model framework named RGPS(Role-Goal-Process-Service) [22]. The role represents the abstract characterization of user behaviors and responsibilities within specified organizational context or domain, which can be used to distinguish different user groups. The goal denotes a descriptive statement of intent of a user or an organization, and can be viewed as an objective that the solution under consideration should achieve. The process is defined as a collection of related, structured activities or tasks that achieve a particular business goal. The service is used to denote a kind of Web-based application such as Web service or Web API, which encapsulates certain computing module and can be accessed by certain interface.

The relations among the four elements are shown in Fig. 2. Roles undertake their corresponding role goals, and actors prefer their respective personal goals. Processes can directly or collaboratively achieve goals. Services can realize certain processes. 
Fig. 2 Relations among RGPS

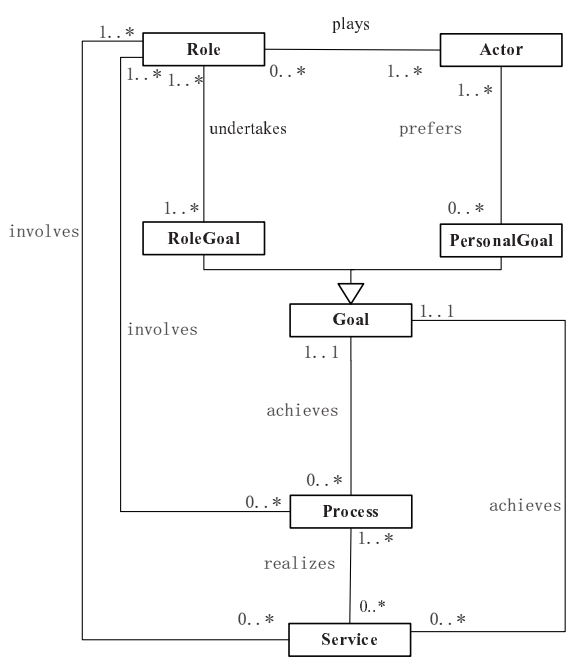

Roles can be involved in the processes and services. In this way, RGPS can be viewed as a hierarchical modeling framework that can contribute to describe and analyze users' requirements, and provide service-based solutions for requirements.

Here, we will briefly introduce the meta-models in RGPS, shown in UML class diagrams. Since roles and goals are closely related, we prefer to model them together. Then, we will introduce the RGPS-based domain modeling, which can be used to created domain models in a specific domain.

\subsection{The Role \& Goal Meta-model}

The role \& goal meta-model is shown in Fig. 3. In [14], the definition of roles was described as: 1) a role is a property assigned to humans that can change dynamically, and 2) humans can have multiple roles simultaneously. In [2], a role was used as a definitive factor in task or service selection, and two kinds of roles were presented, i.e., social role and task role. In RGPS, the roles are mainly used to distinguish different user groups. Users who play the same role are likely to share similar business goals and behavior patterns. Once a user's role in a certain context is recognized, the services closely related to the role may be considered to be recommended to the user.

A role can be played by different actors.In an organizational context, role goals are the goals that a role is in charge of. Actors also have their personal preferences, and these personal preferences are modeled as personal goals. An organization is composed of a collection of related roles.

Goals can be either functional or nonfunctional. Functional goals describe the functions that a system or a service must achieve, and nonfunctional goals are used 


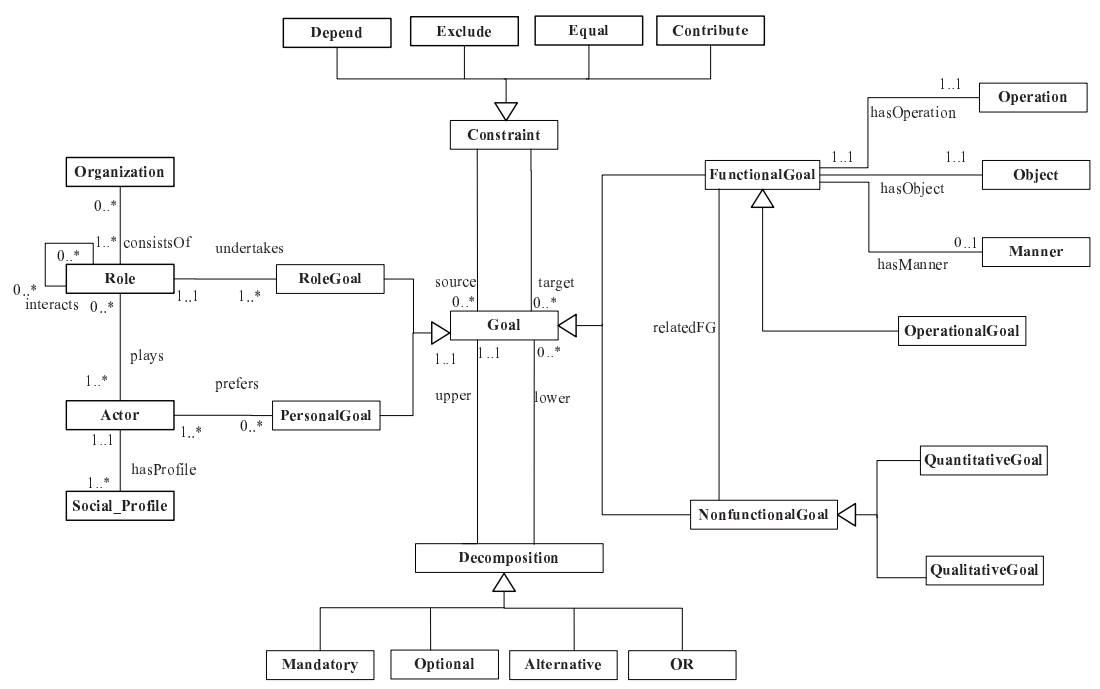

Fig. 3 The role \& goal meta-model

to describe how well the functional goals are exercised. The description of a functional goal consists of three parts: a verb that indicates the operation, a noun that indicates the object dealt with by the operation, and the manner that is either a prefix or a suffix to indicate how the operation affects the object. Each operation can be annotated by a verb concept of domain ontology. Similarly, each object can be annotated by a noun concept of domain ontology. Nonfunctional goals can be classified into quantitative goals and qualitative goals according to their description manner.

Usually, a goal is a high-level statement when it is first proposed, and needs to be decomposed in order to get a concrete and operational description [6,10]. Goal decomposition or refinement depicts how an upper goal can be decomposed into lower goals, and the process will not stop until all the leaf-level sub-goals are operational goals. Each operational goal is the goal that can be directly achieved by an atomic process in the process model. The feature decomposition strategy in FODA (Feature-Oriented Domain Analysis) [7] is adopted during the goal refinement process to support the variability modeling. The decomposition relations between an upper goal and a lower goal set can be classified into four types:mandatory, optional, alternative, and $O R$. When an upper goal $g$ is selected by a user, the lower goal set that has the mandatory relation with $g$ must also be selected; the lower goal set that has the optional relation with $g$ may be or not be selected; exactly one goal from the lower goal set that has the alternative relation with $g$ must be selected; and at least one goal from the lower goal set that has the $O R$ relation with $g$ must be selected.

In addition, the constraint relation between goals can be classified into depend, exclude, equal, and contribute relations. The depend relation means that the realization of a goal depends on the realization of another one; the exclude relation 


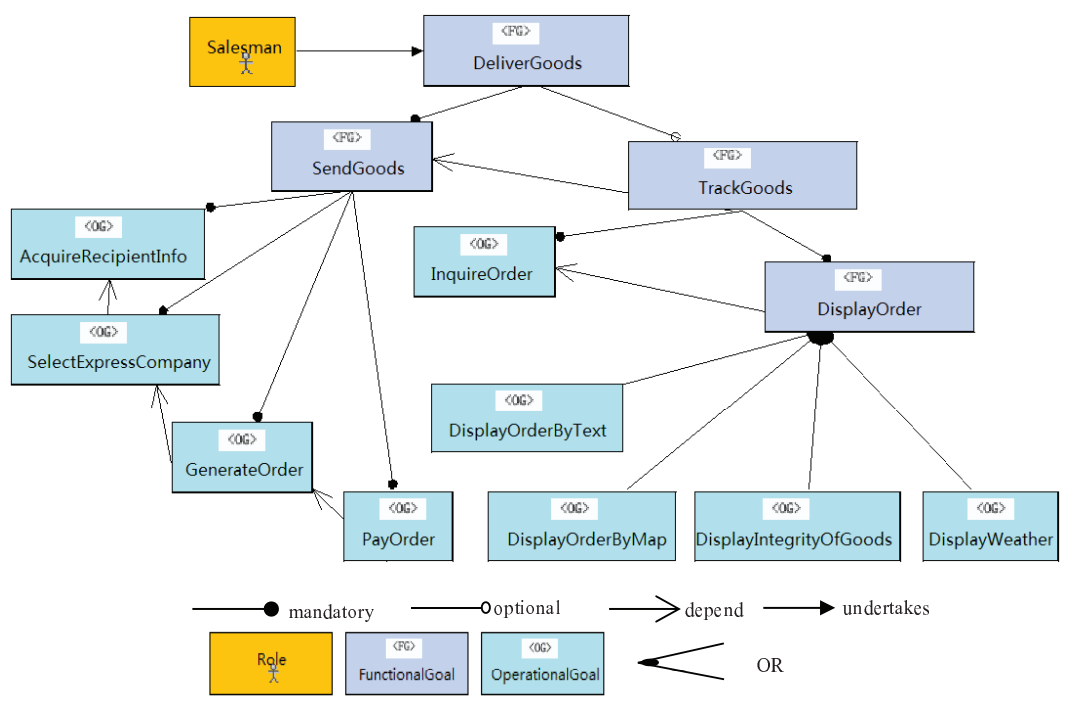

Fig. 4 A role \& goal model

means that two goals cannot be satisfied simultaneously; the equal relation means that two goals are the same in semantics; and the contribute relation means that the realization of a goal can contribute to the realization of another one.

Fig. 4 shows an example of how the role \& goal meta-model can be used to describe a role \& goal model. The model comes from the motivating example in Sect. 2.1. The role goal of the role "Salesman" is "Deliver Goods," which is composed of a mandatory sub-goal "Send Goods" and an optional sub-goal "Track Goods." "Send Goods" can be further refined into four mandatory sub-goals: "Acquire Recipient Info," "Select Express Company," "Generate Order" and "Pay Order." "Track Goods" can be refined into two mandatory sub-goals: "Inquire Order" and "Display Order." The goal "Display Order" can be further decomposed into four $O R$ sub-goals: "Display Order by Text," "Display Order by Map," "Display Integrity of Goods" and "Display Weather." Note that all the leaf-level goals are operational goals, while other goals are functional goals. Moreover, five dependences exist between five pairs of goals: between "Select Express Company" and "Acquire Recipient Info," between "Generate Order" and "Select Express Company," between"Pay Order" and "Generate Order," between "Track Goods" and "Send Goods," and between "Display Order" and "Inquire Order."

\subsection{The Process Meta-model}

The process meta-model is shown in Fig. 5. A process can be either an atomic process or a composite process. The difference between them is that the composite 


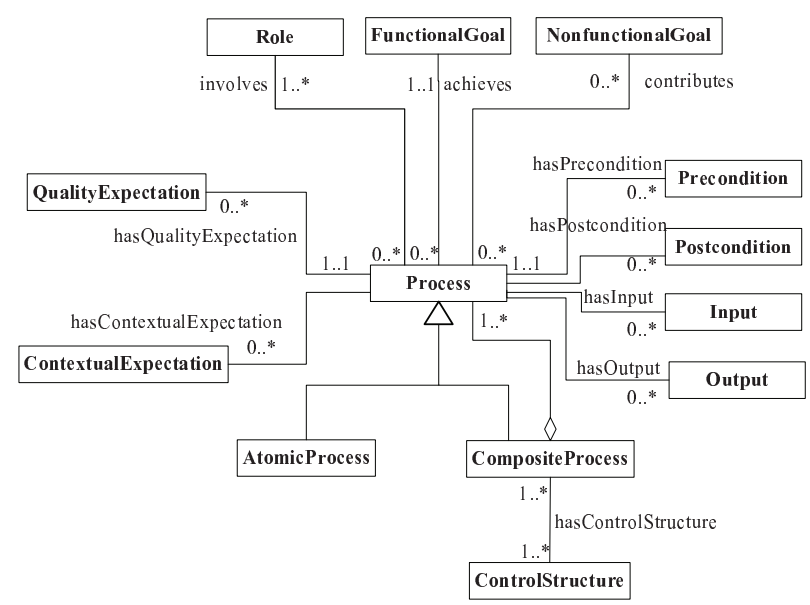

Fig. 5 The process meta-model

process consists of at least one control structure. Five typical control structures are defined: sequence, loop, choice, split-join and any order.

A process can achieve certain functional goals, and contribute to some nonfunctional goals. A process has four basic attributes: input, output, precondition and effect. Input and output represent the data flow transformed by the process. Precondition and effect indicate the constraints to be held before the process starts and after the process is normally finished, respectively.

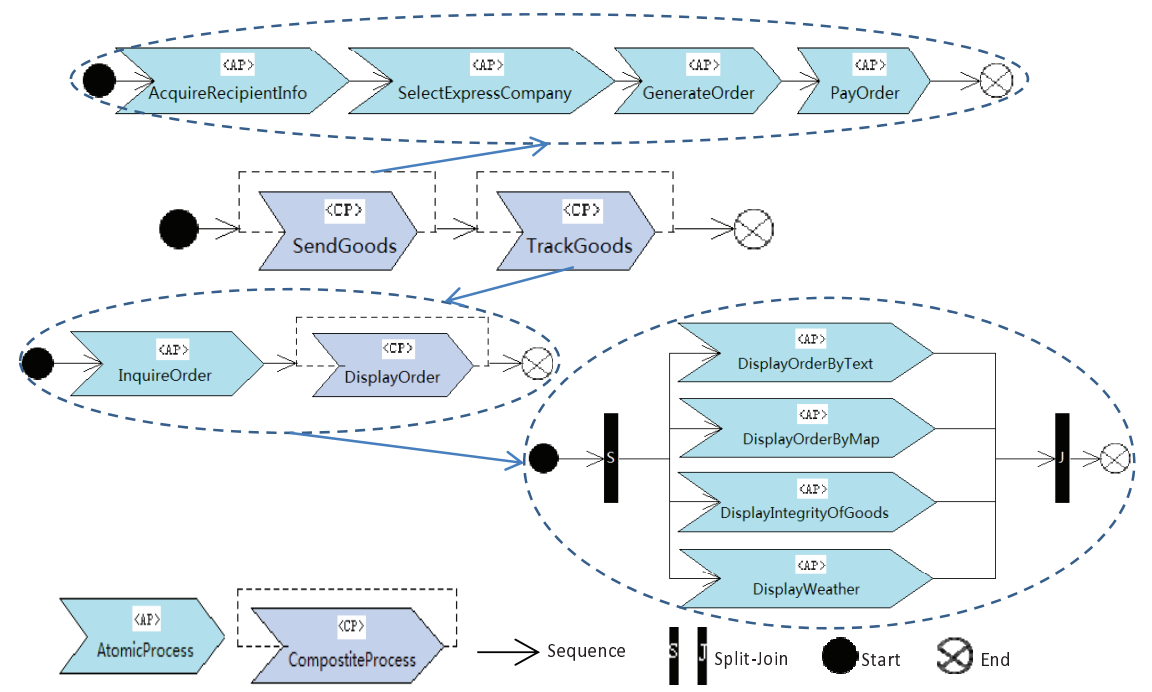

Fig. 6 A process model 
In addition, a process may have nonfunctional expectations including quality expectation and contextual expectation. The former describes the quality requirements (e.g., the range of the response time); the latter describes contextual requirements (e.g., the range of the distance to the office) of the process.

Fig. 6 illustrates how the process meta-model can be used to describe a process model. We use the modeling notations adapted from [1] to represent the process model. The process model consists of two composite processes "SendGoods" and "TrackGoods," connected by control structure sequence. The details of these composite processes are shown in the dashed ellipses. "SendGoods" consists of four atomic processes connected by control structure sequence. "TrackGoods" consists of an atomic process "InquireOrder" and a composite process "DisplayOrder" connected by control structure sequence. The composite process "DisplayOrder" consists of four atomic processes connected by control structure split-join.

\subsection{The Service Meta-model}

As shown in Fig. 7, the service meta-model is used to capture the common semantics of services. Typical services description languages include WSDL [19], OWL$\mathrm{S}$ [13], and WSMO [8].

A service has input message and output message, which can be constrained by precondition and postcondition, respectively. Precondition is used to specify the state that shall have to satisfied before a service is invoked, while postcondition is used to specify the state that have to be satisfied after a service is invoked successfully. In order to add semantic information for a service, the input message and output message can be annotated by concepts of domain ontology. The nonfunctional semantics of a service is depicted by quality property and contextual prop-

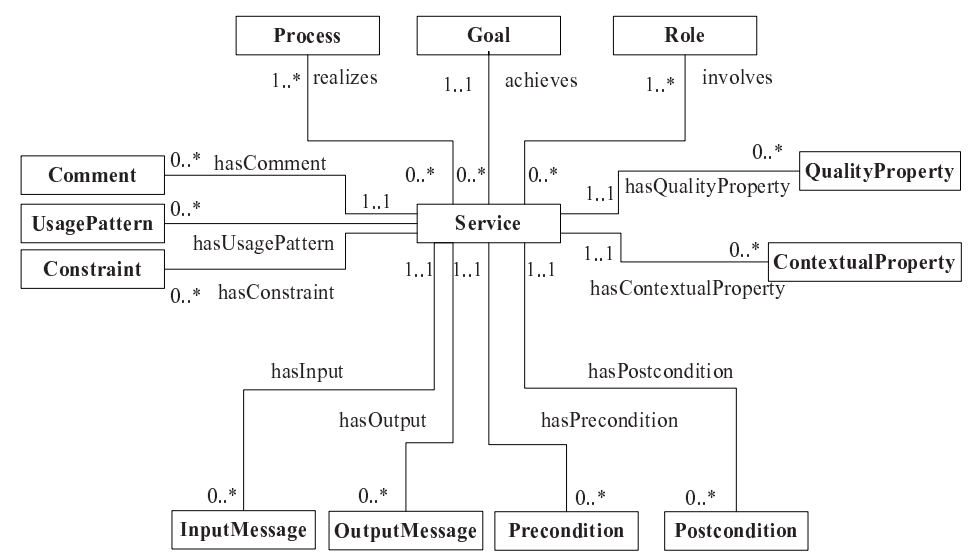

Fig. 7 The service meta-model 
erty, which can be used to represent the value of service in certain aspects such as response time, cost, and contextual restriction. These two kinds of properties can be used to match against the quality expectation and contextual expectation of processes, respectively.

As an embodiment of Web 2.0, a service can be annotated by comments or social tags, which are created by the people using the service. The goal that is used to show the objectives of the service, and the role that denotes the beneficiary of the service, can be provided by service creators. The usage patterns and constraints can be provided by service providers.

We will use an example of payment service mentioned in Sect. 2.1 to illustrate how the service meta-model can be used to describe a service model. The input messages of the service are card_ID, card_PIN, and transaction_amount, while the output message is a boolean variable transaction_result. The precondition of the service is that the input card_ID and card_PIN have to be valid and the balance of the card is greater than the value of the input transaction_amount. The postcondition is that the payment is charged and the balance of the card is decreased after the transaction is completed. The constraint of the service is that if no operation is received from users for 15 minutes during the execution process of the service, the connection will be expired for security reasons. The usage pattern indicates that the service is usually used followed by a shipping service if a merchandize is involved. The quality property denotes that the security of the service is guaranteed. The contextual property denotes that the service is only available in China. The comments can be ad hoc fragmental documents such as social tags and user comments (e.g., good user experience and free of charge).

\subsection{Domain Modeling}

Based on the RGPS meta-model framework, we propose an ontology-based domain modeling approach named O-RGPS. O-RGPS aims to provide a method to construct and manage domain models according to common requirements in a specific domain, which can be reused in requirements analysis and process customization.

In O-RGPS, domain ontologies including domain entity ontology and domain operation ontology are the basis for domain modeling, which can provide semantic annotation for domain models. Based on the domain ontologies, domain models can be created following the RGPS meta-models. More specifically, this approach can be generalized as the following steps:

1. Construct domain entity ontology and domain operation ontology. The domain entity ontology describes entity (noun) concepts and relationships among them, while domain operation ontology describes the operation (verb) concepts and relationships among them.

2. Construct role \& goal models and annotate the models using domain ontologies. A role \& goal model can be described in OWL and stored into domain model repository. 
3. Construct process models and annotate the models using domain ontologies. A process model can be described in extended OWL-S and stored into domain model repository.

4. Construct service models and annotate the models using domain ontologies. Besides the information extracted from service description files such as WSDL, some other information will be modeled as attributes of the service including the role that closely related to the service, the goal that the service can achieve, and the process that the service can realize. A service model can be described in extended OWL-S and stored into domain model repository.

5. Classify and group all the domain models according to different domain problems they are related to, and create the relations among domain models in a group so as to improve the efficiency of querying models.

\section{RGPS-based Process Customization Approach}

The approach of requirements driven process customization will be introduced in detail in this section.

\subsection{Procedure of Customization}

Based on the domain models created according to the common requirements in a specific domain, users' personalized requirements can be analyzed and corresponding customized service-oriented solutions will be constructed. As shown in Fig. 8, the following steps are used to complete the procedure: (1) elicit users' requirements; (2) match the requirements with domain goals; (3) supplement and refine goals; (4) configure processes; and (5) discover services.

Users' requirements can be expressed in two ways. In the first way, the requirements can be represented in SORL, a pseudo natural language to be introduced in Sect. 4.2. Then users' requirements can be elicited and parsed, and the output will become the initial goals. The other way is to define some domain requirements templates for users to select from. That is, users can browse all domain models in templates and manually choose the domain, role, and goal information that they prefer step by step. For the initial goals, we need to match them with domain goals in Step 2. If the goals can be matched, it will go to Step 3 for further supplement and refinement. Otherwise, the unmatched goals will be returned to users to revise their requirements. Once the goal model is refined, we will go to Step 4 to configure processes by leveraging the mapping between the goal model and process model. It is worth noting that the process configuration in this chapter mainly refers to removing, adding or modifying process model elements to meet the given requirements. When the process models are customized, we need to discover services from our service supermarket or publicly accessible service registries based on the process 


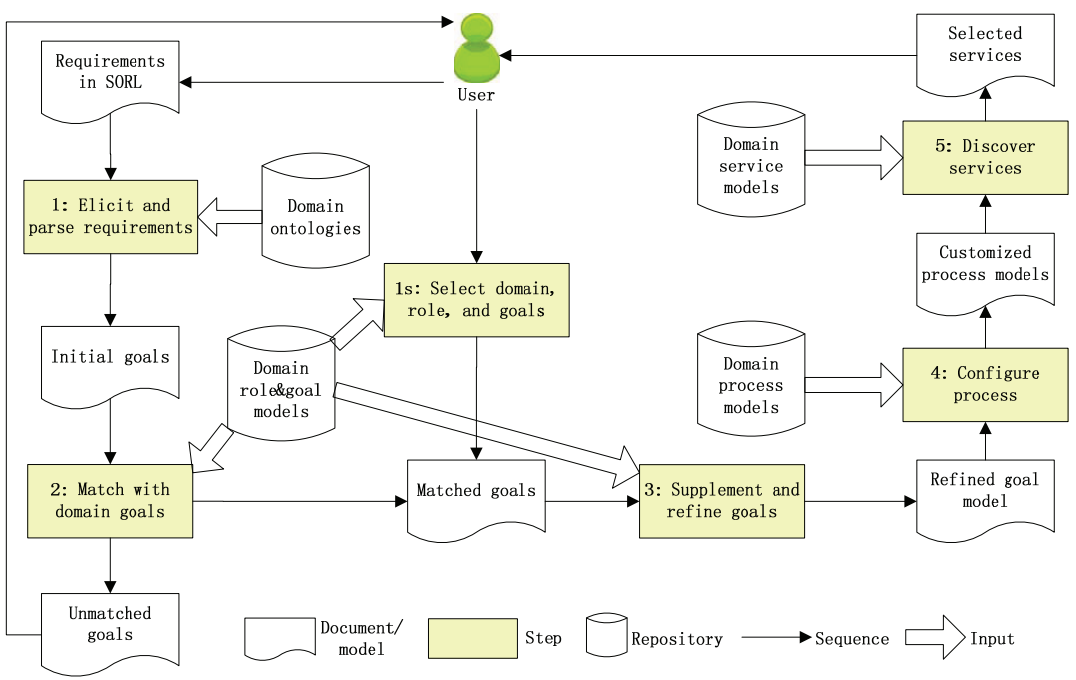

Fig. 8 Procedure of RGPS-based process customization

models. Finally, the selected services will be returned to users. Note that users will be involved in Steps 3, 4, and 5.

\subsection{Elicit users' requirements}

Based on the natural language patterns and RGPS, a requirements elicitation language named Service Oriented Requirements Language (SORL) [12] has been designed for eliciting users' requirements. In SORL, two kinds of sentence patterns are defined: domain-independent sentence pattern and domain-specific sentence pattern. We have defined 16 domain-independent sentence patterns that cover the description of functional goals, nonfunctional goals, and so on. For example, the sentence "the salesman will deliver goods to his customer" belongs to the functional pattern. Here, we just list part of them.

Domain-independent Pattern $::=$ Functional Pattern $\mid$ NonFunctional Pattern;

Functional Pattern $::=$ [Role] Verb Noun;

NonFunctional Pattern $::=$ Qualitative Description Pattern $\mid$ Quantitative Description Pattern;

Qualitative Description Pattern $::=$ Noun be Adjective;

Quantitative Description Pattern $::=$ Noun Comparison-Operator Number [Unit]; . .

The domain-independent sentence patterns are defined according to the role \& goal meta-model. The domain-specific sentence pattern is summarized from the characteristics of the domain-specific expressions, which are varied among different domains. 
Following these sentence patterns in SORL, users' requirements can be expressed. Firstly, some natural language processing techniques, such as segmentation and stemming, are applied to normalize the requirements text. Next, several steps are taken: the requirements text will be tagged by the grammatical item in SORL; the sentence patterns can be recognized based on the finite state automata; and the roles and goals can be extracted based on the mapping between SORL grammatical item and elements in the role \& goal meta-model. In this way, an initial goal list can be identified.

Another candidate way is for users to manually select related elements from domain models according to a series of requirements templates. Providing a series of requirements elicitation templates, users can select a domain from the first template that lists all domains. Afterwards, the user can select a role that he hopes to play in the domain from the second template that lists all possible roles in the selected domain. The goals related to the role will then be listed in the third template, and the user can select the goals from the goal list. With selected specific domain goals, the analysis procedure can move to the goal refinement (Step 3).

\subsection{Match with domain goals}

In this step, the goals in the initial goal list will be matched with the goals in domain goal models. The goal-matching problem can be solved by calculating the semantic similarity of the two goals.

The similarity between two goals is calculated based on ontology-based concept similarity, which has been widely investigated [20]. Recall that a goal consists of two mandatory attributes: operation and entity (object). The similarity between two goals thus consists of two parts: the similarity of two operation concepts and the similarity of two entity concepts. The similarity of two operation concepts can be calculated using Formula (1). Depth $\left(O_{1}\right)$ means the length of the path from concept $O_{1}$ to the root of the ontology. $C\left(O_{1}\right)$ denotes the number of descendants or hyponymy concepts of concept $O_{1}$. $C\left(O_{1}\right) \cap C\left(O_{2}\right)$ denotes the number of the intersection of descendants of concepts $O_{1}$ and $O_{2}$. Distance $\left(O_{1}, O_{2}\right)$ denotes the number of edges that link concepts $O_{1}$ and $O_{2}$ in the domain ontology hierarchy. The principles in Formula (1) are as follows. If the semantic overlap between two concepts is higher, then the two concepts are more similar; if the distance of two concepts is larger, then the two concepts are less similar; if the difference between the depth of two concepts is larger, then the two concepts are less similar. Similarly, the similarity of two entity concepts $E_{1}$ and $E_{2}$ can be calculated in Formula (2).

OperationSim $\left(O_{1}, O_{2}\right)=\frac{C\left(O_{1}\right) \cap C\left(O_{2}\right)}{\left(\operatorname{Distance}\left(O_{1}, O_{2}\right)+1\right) \times\left(\left|\operatorname{Depth}\left(O_{1}\right)-\operatorname{Depth}\left(O_{2}\right)\right|+1\right)}$ 


$$
\operatorname{EntitySim}\left(E_{1}, E_{2}\right)=\frac{C\left(E_{1}\right) \cap C\left(E_{2}\right)}{\left(\operatorname{Distance}\left(E_{1}, E_{2}\right)+1\right) \times\left(\left|\operatorname{Depth}\left(E_{1}\right)-\operatorname{Depth}\left(E_{2}\right)\right|+1\right)}
$$

To calculate the similarity of an initial goal $G_{i g}$ and a domain goal $G_{d g}$, the similarity of operation concepts and the similarity of entity concepts should be combined. Formulas (3) and (4) show how to calculate the proportions of the operation part and the entity part, respectively. Note that $O_{d g}$ and $E_{d g}$ denote the operation concept and entity concept of domain goal $G_{d g}$, respectively. The principle in Formula (3) is as follows. If a concept lies in a deeper layer of an ontology, then it will have more specific semantics, and is closer to the meaning of the user' requirements.

$$
\begin{aligned}
\text { OProportion } & =\frac{\operatorname{Depth}\left(O_{d g}\right)}{\operatorname{Depth}\left(O_{d g}\right)+\operatorname{Depth}\left(E_{d g}\right)} \\
\text { EProportion } & =\frac{\operatorname{Depth}\left(E_{d g}\right)}{\operatorname{Depth}\left(O_{d g}\right)+\operatorname{Depth}\left(E_{d g}\right)}
\end{aligned}
$$

To sum up, the goal similarity of $G_{i g}$ and $G_{d g}$ can be calculated in Formula (5). Considering the range of the goal similarity should be within $(0,1)$, Formula (6) is used to normalize the result of Formula (5), where $\beta$ is a factor whose value is between 0 and 1 .

$$
\begin{aligned}
& \operatorname{GoalSim}\left(G_{i g}, G_{d g}\right)^{\prime}=\text { OProportion } \times \text { OperationSim }\left(O_{i g}, O_{d g}\right) \\
& + \text { EProportion } \times \text { Entity } \operatorname{Sim}\left(E_{i g}, E_{d g}\right) \\
& \operatorname{GoalSim}\left(G_{i g}, G_{d g}\right)=1-\beta^{\operatorname{GoalSim}\left(G_{i g}, G_{d g}\right)^{\prime}}
\end{aligned}
$$

In this way, the initial goals can be matched with the goals in domain goal models according to their similarity. For the unmatched initial goals, two actions will be taken. First, the user can manually browse domain goals according to Step 1s. Second, the unmatched goals will be marked and recorded for further analysis by domain engineers to check whether the domain models should evolve.

\subsection{Supplement and refine goals}

According to the RGPS meta-model framework, each goal is associated with a role. Therefore, based on the goals that have matched with domain models, we can determine the role that the user plays. Since users' requirements are usually incomplete, all the goals related to the specific role will be listed for users to select. In this way, more complete relative goals may be identified.

As mentioned before, a goal can be decomposed into more concrete sub-goals according to the hierarchical structure in domain goal model. When a goal list is obtained, we need to check whether the decomposition and constraint relations are 
satisfied towards their semantics in domain goal models. Examples are alternative, mandatory, depend, and exclude. For the mandatory relation, we need to check whether all goals in the lower goal set have been selected. For the alternative relation, we need to check whether one and only one goal from the lower goal set is selected. For the depend relation, the goals depended by the selected goals will also be added to supplement users' requirements. For example, if a user has selected a goal "book airline ticket by credit card" that has the depend relation with the goal "validate credit card," then the latter must be selected. If two goals that have the exclude relation exist in users' selected goal list, then one of them must be removed. Since an operation might cause a new problem, the checking process should be iterated until the semantics of all decomposition and constraint relations are satisfied.

\subsection{Configure processes}

The configuration of processes will be based on users' operations on the refined goal model. Here we discuss the mapping between the goal model and process model. Three kinds of mappings are defined as shown in Table 1: mapping between goals and processes (Row 1-2), mapping between the relations in the goal model and the control structures in the process model (Row 3-7), as well as the nonfunctional properties between goals and processes (Row 8).

The operational goal cannot be further decomposed, which is similar to the atomic process in the process model. Therefore the operational goal can be mapped into the atomic process, while the functional goals can be mapped into the composite process. The goal decomposition relations are used to connect an upper goal and a lower goal set. Since the relations mandatory and optional can coexist in the decomposition of an upper goal, the related goals in the lower goal set are independent with each other, which indicate that their corresponding processes can be achieved in any order. In other words, the two relations can be both mapped into the control structure any order. For the relation alternative, one and only one lower goal can be selected, which is similar to the control structure choice. For the relation $O R$, at least one lower goal should be selected into the resulting goal set, which is compa-

Table 1 Mapping between the goal model and process model

\begin{tabular}{ll}
\hline Elements in Goal Model & Elements in Process Model \\
\hline Functional Goal & Composite Process \\
Operational Goal & Atomic Process \\
mandatory & any order \\
optional & any order \\
alternative & choice \\
OR & split-join \\
depend & sequence \\
relatedFG & expectation of a process \\
\hline
\end{tabular}


Table 2 Mapping between the operations on the goal model and process model

\begin{tabular}{|c|c|}
\hline Users' Operations on Goal Model & Configuration of Process Model \\
\hline Select or deselect optional sub-goals & $\begin{array}{l}\text { Add or delete sub-processes within the control structure } \\
\text { any order }\end{array}$ \\
\hline Select or deselect $O R$ sub-goals & $\begin{array}{l}\text { Add or delete sub-processes within the control structure } \\
\text { split-join }\end{array}$ \\
\hline Reselect an alternative sub-goal & $\begin{array}{l}\text { Delete sub-processes within the control structure choice } \\
\text { or modify the condition of choice }\end{array}$ \\
\hline $\begin{array}{l}\text { Add a functional goal that has depend } \\
\text { relation with existing goals }\end{array}$ & $\begin{array}{l}\text { Add a process connected with an existing process using } \\
\text { the control structure sequence }\end{array}$ \\
\hline $\begin{array}{l}\text { Add a functional goal that does not have } \\
\text { depend relation with existing goals }\end{array}$ & $\begin{array}{l}\text { Add a process connected with existing processes using } \\
\text { the control structure any order }\end{array}$ \\
\hline Delete functional goals & Delete corresponding processes \\
\hline Add or delete nonfunctional goals & Add or delete the expectation properties of a process \\
\hline
\end{tabular}

rable to the control structure split-join. In addition, the relation depend means that achieving a goal should depend on the achievement of another one, which is similar to the control structure sequence. As discussed earlier, a nonfunctional goal can take effect on functional goals, which is similar to the process's constraints defined by the expectations.

The relations mandatory, optional, alternative, and $O R$ belong to the vertical decomposition relations, while the relation depend belongs to the horizontal relation. When there is a crossover between a vertical relation and a horizontal relation, the horizontal relation has a higher priority. For example, assume both goals $A$ and $B$ are mandatory goals, and $B$ depends on $A$. According to the mapping rule in Row 3 of Table 1, their corresponding processes will be connected by control structure any order. However, according to the mapping rule in Row 7 of Table 1, their corresponding processes will be connected by sequence. In this case, the control structure sequence will be selected since the relation depend has a higher priority.

Following the mapping between the goal model and process model, we discuss the configuration of process models based on the operations on goal models, as shown in Table 2.

1. Select or deselect optional sub-goals

As shown in Fig. 9(a), the optional sub-goals can correspond to the processes P1, $\cdots, \mathrm{Pn}$, within the control structure any order. If a user only selects the sub-goals that correspond to $\mathrm{Pi}$ and $\mathrm{Pj}$, then the result is shown in Fig. 9(b). If only one goal is selected, then the control structure any order will be deleted, and only one process is left, as shown in Fig. 9(c).

2. Select or deselect $O R$ sub-goals

As shown in Fig. 10(a), the OR sub-goals can correspond to the processes P1, $\ldots, \mathrm{Pn}$, within the control structure split-join. If a user only selects the sub-goals that correspond to Pi and Pj, then the result is shown in Fig. 10(b). If only one goal is selected, then the control structure split-join will be deleted and only one process is left, as shown in Fig. 10(c). 
Fig. 9 Process customization for optional sub-goals

Fig. 10 Process customization for $O R$ sub-goals

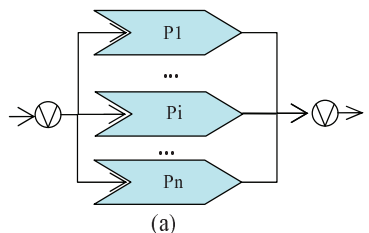

(a)

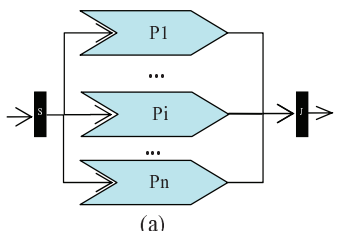

(a)
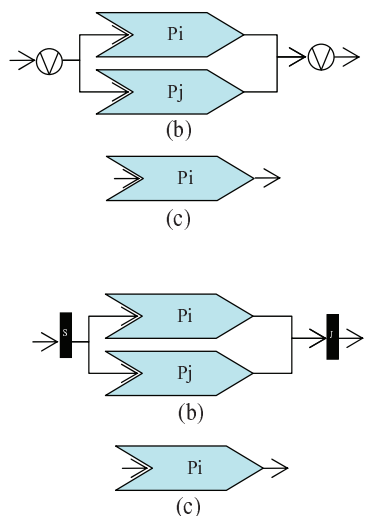

3. Reselect an alternative sub-goal

As shown in Fig. 11(a), the alternative sub-goals can correspond to the processes $\mathrm{P} 1, \cdots, \mathrm{Pn}$, within the control structure choice. If a user selects the sub-goal that corresponds to $\mathrm{Pi}$, then the other sub-processes within control structure choice as well as the choice itself should be deleted, as shown in Fig. 11(b).

Fig. 11 Process customization for an alternative subgoal

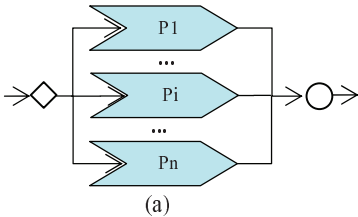

(a)

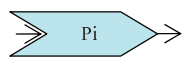

(b)

4. Add a functional goal with the depend relation

According to Table 1, the relation depend in the goal model corresponds to the control structure sequence in the process model. Fig. 12(a) shows the original process Pi. If the added functional goal $\mathrm{Gj}$ depends on the goal that Pi can achieve, then the process $\mathrm{Pj}$ that achieves $\mathrm{Gj}$ will be connected with Pi using the control structure sequence, as shown in Fig. 12(b). Note that in this example, we use the operation goal to represent the functional goal.

Fig. 12 Process customization for adding a functional goal with depend relation
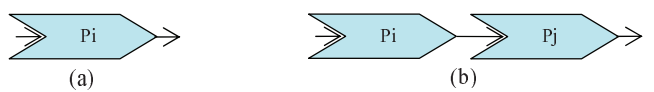

\section{Add a functional goal without the depend relation}

Fig. 13(a) shows the original process $\mathrm{Pi}$, which might be an atomic process or a composite process. If the added functional goal $\mathrm{Gj}$ does not depend on any goal that $\mathrm{Pi}$ can achieve, then the process $\mathrm{Pj}$ that can achieve $\mathrm{Gj}$ will be connected with 
Pi using the control structure any order, as shown in Fig. 13(b). Note that in this example, we use the operation goal to represent the functional goal.

Fig. 13 Process customization for adding a functional goal without depend relation

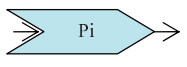

(a)

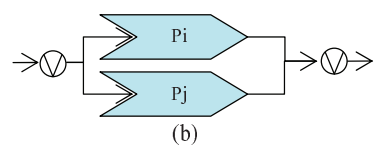

6. Delete functional goals

When the functional goals are deleted, we just need to delete the corresponding processes that can achieve the functional goals.

7.Add or delete nonfunctional goals

According to Table 1, nonfunctional goals correspond to the nonfunctional expectations of a process. When nonfunctional goals are added or deleted, the nonfunctional expectations of the corresponding process will be added or deleted accordingly.

\subsection{Discover services}

After the process models are configured according to users' requirements, the final step is to discover services that can collaboratively realize the processes. During service discovery, two kinds of service registries are considered: our service supermarket and publicly accessible service registries.

We have developed a service supermarket based on the service meta-model in RGPS. When a service is registered in the service supermarket, besides the information extracted from the WSDL files such as input message and output message, other information can also be registered. Examples include the role that is closely related to the service, the goal that the service can achieve, and the process that the service can realize. Since such additional information is not a mandatory property of services, if they are available, we could use role matching, goal matching or process matching to find corresponding services. Otherwise, we have to make a matchmaking based on the input and output information, as well as the nonfunctional expectations to find corresponding services.

For the publicly accessible service registries such as ProgrammableWeb, we have developed a service categorization method enhanced by incrementally enriched domain knowledge [23]. Using this method, the services in the registry can be classified into different domains. Therefore, the services belonging to the same domain with the proposed requirements will be matched with the processes, which can shorten the searching space of services. The matching is conducted by comparing the similarity between the description of a service and a process based on domain ontology. The service whose similarity with the process is above a given threshold can be selected for further consideration. 
In this way, the selected services can be orchestrated according to the customized process model.

\section{The Visualization Prototypes}

To support our approach, we have developed two visualization prototypes: a domain modeling tool and a service supermarket.

\subsection{O-RGPS Domain Modeling Tool}

The O-RGPS domain modeling tool is a plug-in tool, which is similar to the UML modeling tool in Eclipse. It is developed using $\mathrm{GEF}^{4}$ (Graphical Editor Framework) and $\mathrm{EMF}^{5}$ (Eclipse Modeling Framework).

By using the tool, three kinds of domain models can be created including role \& goal models, process models, and service models. Fig. 13 shows a snapshot of goal modeling. By dragging the icons listed in the palette into the editing area, domain

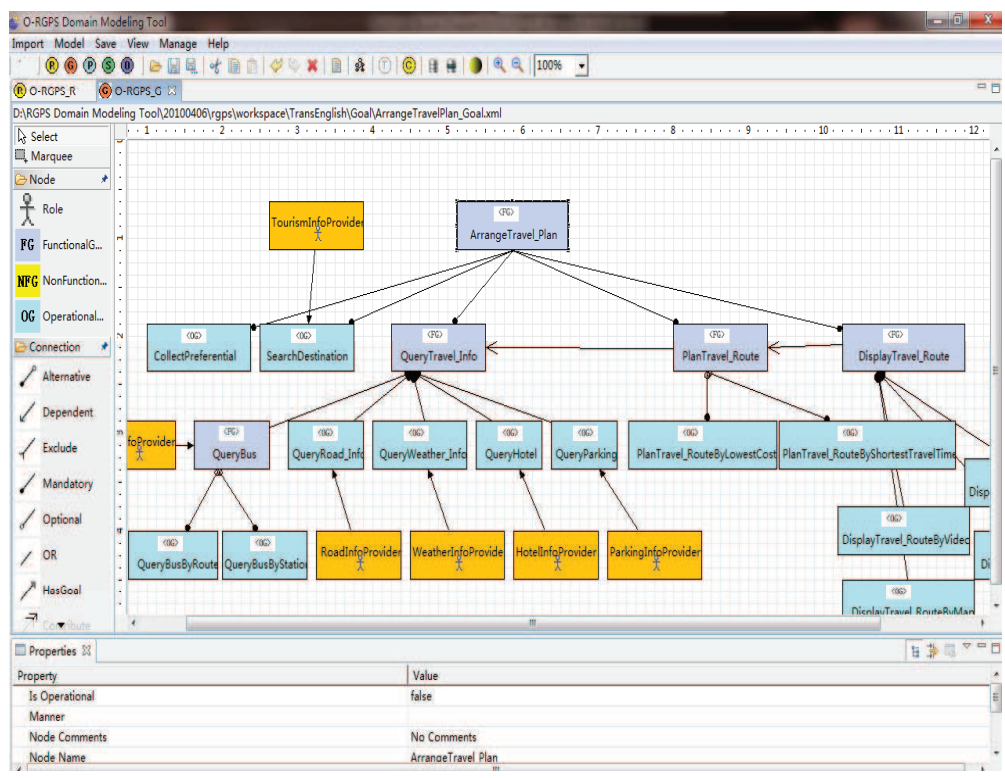

Fig. 14 Snapshot of goal modeling interface

\footnotetext{
${ }^{4} \mathrm{http} / / / \mathrm{www} . e c l i p s e . o r g / g e f /$

${ }^{5}$ http://www.eclipse.org/modeling/emf/
} 
engineers can create the goal nodes and their relations. They can also set the properties of the goals by editing the "Properties" tab in the part below. The tool also provides a function of flexible reusing goal models in different granularity. Furthermore, if a goal model is properly created, a process model can be automatically transformed, which can greatly alleviate the modeling burden of domain engineers. Our tool can provide semantic support for domain models by importing specific domain ontologies and annotating certain elements of domain models by concepts of domain ontology.

\subsection{The Service Supermarket CloudCRM}

The service supermarket we have developed is named as CloudCRM ${ }^{6}$ since the original services are encapsulated from an open-source software called SugarCRM in the CRM domain. The basic idea of the service supermarket is similar to a supermarket where users can select their goods and receive some recommended products. The service supermarket is more than a service registry that acts as a centralized yellow page to help users find related services [24], since the multi-tenancy architecture is also designed in the supermarket.

\subsubsection{Services encapsulation in service supermarket}

As mentioned before, the original services are encapsulated from SugarCRM that is composed of 14 functional modules. Since SugarCRM is developed using PHP, we firstly create a .php file for each functional module by using SugarCRM API or extracting codes directly from the open-source codes; and then we leverage a tool named Zend Studio for Eclipse to create a .wsdl file from a .php file. Using such a procedure, we have encapsulated some CRM services, such as accounts service, targetLists service, and contact service.

During service encapsulation, multi-tenancy design of these services is also supported. Multi-tenancy is defined as the ability of an application to provide the same service to different tenants [4]. In CloudCRM, separated database is chosen as the solution of data isolation, which means that each tenant will have their separate databases.

Besides the services encapsulated from SugarCRM, we have also registered many Web services into our service supermarket, such as weather forecast service, map service, and flight query service. The services are registered in CloudCRM according to the RGPS meta-model framework, which help improve the precision and efficiency of service query.

\footnotetext{
${ }^{6}$ http://cloud.whu.edu.cn:8080/En_CloudCrm/login.jsp
} 


\subsubsection{Users in the service supermarket}

Since multi-tenancy is an important feature in CloudCRM, users can play three kinds of roles according to their assigned permissions: system administrator, tenant administrator, and end user. The system administrator can manage tenant administrators, while the tenant administrator can manage end users.

For tenant administrators who are the representatives of each organization in CloudCRM , they are responsible for managing the end users in their organizations as well as their subscribed services. More specifically, they can select appropriate services for their organizations; they can allocate available services for each user according to the business role that the user will play; they can also pause, cancel, and restore the right of an end user to use certain services.

For end users, they are allowed to use the authorized services allocated by the tenant administrators; they can also propose their requirements to use more services. CloudCRM provides a collection of requirements templates to help elicit users' requirements and recommend proper services.

\section{A Case Study}

In this section, we will use the motivating example introduced in Sect. 2.1 to show how it can be resolved by our approach on top of the CloudCRM service supermarket.

In our approach, a user's requirements can be defined in two ways. One way is to define the requirements in Service Oriented Requirements Language (SORL). The other way is to configure user requirement templates (i.e., domain, role and goal)

\section{RGPS-based Process Customization}

\begin{tabular}{|c|c|c|}
\hline Selected Domain: & \multicolumn{2}{|r|}{ CRM } \\
\hline Selected Role: & \multicolumn{2}{|c|}{ Salesman } \\
\hline Please Select Goal: & \multicolumn{2}{|c|}{ 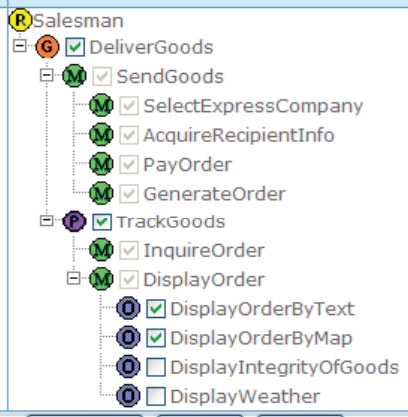 } \\
\hline & Previous & $\begin{array}{lll}\text { Next Submit } & \\
\end{array}$ \\
\hline
\end{tabular}

Fig. 15 Goal refinement in CloudCRM 


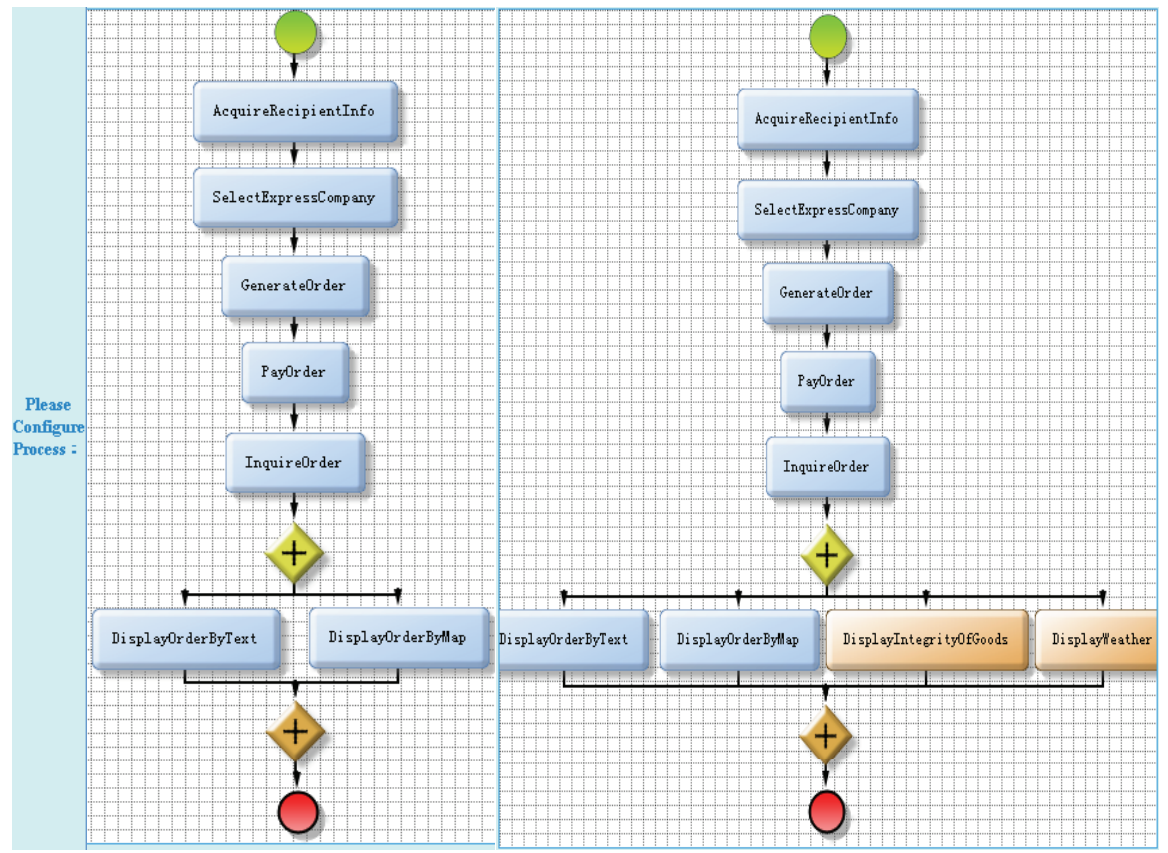

Fig. 16 Process configuration in CloudCRM

created based on domain models. Fig. 15, generated by our system according to the domain goal model shown in Fig. 4, shows a goal refinement scenario when a user selects the goal "Deliver Goods" as a salesman. During the goal refinement process, the semantics of the decomposition relations have to be satisfied, which will be judged and verified in real time by our system. After the user finishes goal refinement, the constraint relations will be checked.

After the goal refinement process, our system will move forward to the process configuration phase. Based on the configured goals, a mapping between the goal model and the available process models will lead to a recommended template as shown in the left picture of Fig. 16. Five atomic processes "Acquire Recipient Info," "Select Express Company," "Generate Order," "Pay Order," and "Inquire Order" are connected by a control structure sequence, followed by the control structure split-join that connects two atomic processes "Display Order by Text" and "Display Order by Map." If the user selects the other two OR sub-goals "Display Integrity of Goods" and "Display Weather" during the goal refinement, the corresponding atomic processes will be added in the control structure split-join, as shown in the right picture of Fig. 16.

Based on the configured process model, some services can be identified from the service supermarket, such as a contact service, Delivery 100 API, Baidu map $\mathrm{API}^{7}$,

\footnotetext{
${ }^{7}$ http://openapi.baidu.com/map/
} 
Check RFID service, and Yahoo! weather $\mathrm{API}^{8}$. The contact service is extracted from SugarCRM, which can support browsing and searching a recipient's information. The Delivery 100 API is an open Web API that can support querying orders of most shipping companies in China. The Check RFID service is a Web service to query the integrity of expensive goods. If all RFID raw data of the goods collected from the RFID readers in every transit station is identical, then the goods is considered to be well maintained during the shipping process. Otherwise, the goods might be destroyed before reaching a certain station. The Baidu Map API can be used to display the route of a transportation in the map. The Yahoo! Weather API can be used to show weather information of the cities in a shipping route.

In summary, such services from the service supermarket can be mashed up with other services developed in house (such as "express company query service") based on the configured process model. The upper picture in Fig. 17 shows a query of a shipping order in China and its results represented in text. Each line in the query result records a shipping status of the goods in a chronological order. For example, the first line shows that the goods arrived at the shipping office on August 25, 2011. The bottom left picture shows the shipping route of the goods as well as the integrity of the order, which alerts the user that the goods might be destroyed before reaching the the city "FuZhou," since the RFID raw data in "FuZhou" and that in its previous site are not identical. The bottom right picture lists the current weather information of all cities in the shipping route.

\section{Conclusions}

Service-oriented process customization is a key issue in SaaS. In this chapter, based on the RGPS meta-model framework, we propose a service-oriented process customization approach that can help end-users configure a personalized mashup in design time. Corresponding visualization prototypes are introduced, and a case study illustrates how to follow our approach to customize a shipping mashup. Our approach depends on the quality of domain models to a large extent. If users' requirements cannot be satisfied, their unmatched goals will be recorded, which will in turn contribute to the evolution of domain models. So our approach is an iterative method in essence.

The RGPS meta-models introduced in this chapter have been accepted as several parts of ISO/IEC 19763, that is, ISO/IEC 19763-8 "Metamodel for role and goal registration," ISO/IEC 19763-5 "Metamodel for process model registration," ISO/IEC 19763-7 "Metamodel for service registration," and ISO/IEC 19763-9 "On demand model selection based on RGPS." Currently, these standards are under development.

Our future research will focus on the following directions. First, we will integrate business rules in process customization. Second, nonfunctional requirements

\footnotetext{
${ }^{8} \mathrm{http} / / /$ developer.yahoo.com/weather/
} 


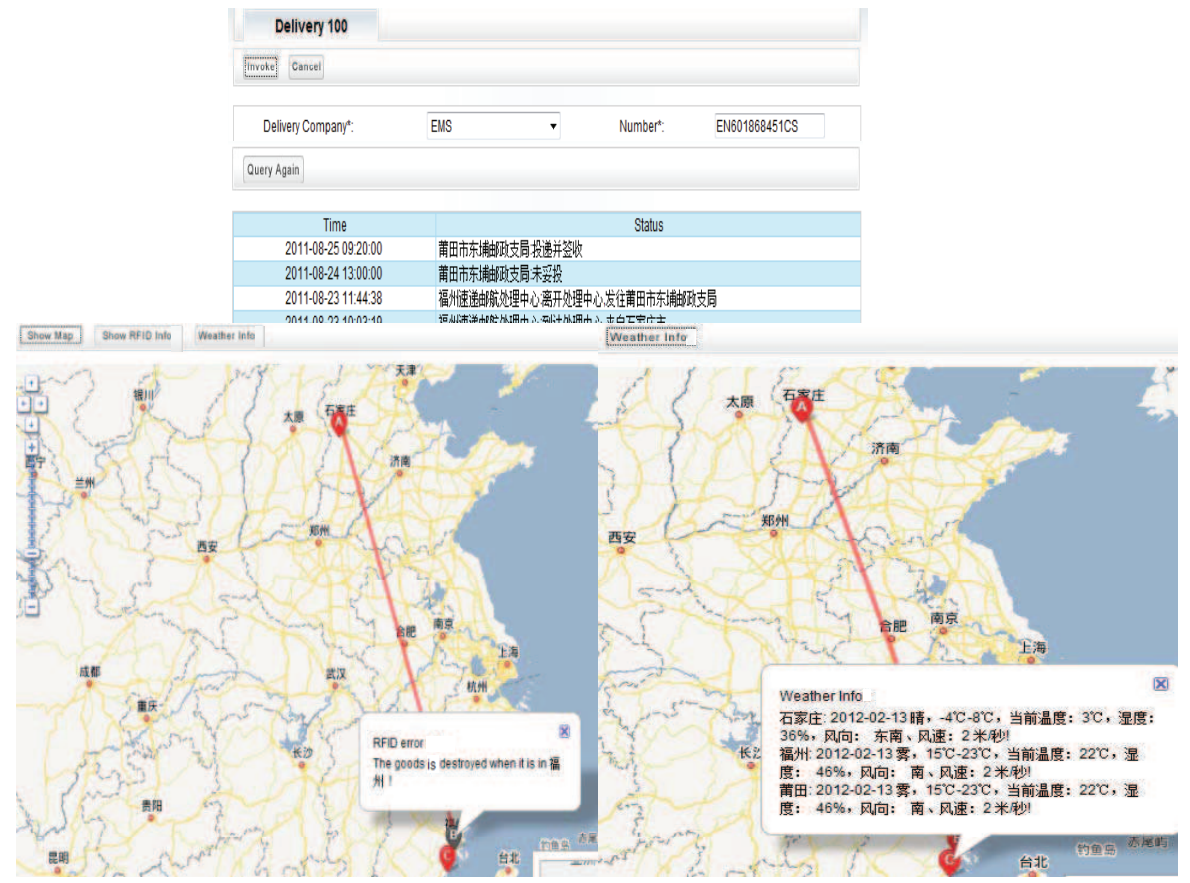

Fig. 17 Created mashup

will be thoroughly investigated in process customization. Finally, the multi-tenancy architecture and the scalability of the service supermarket will be explored.

Acknowledgements The work is partially supported by the National Natural Science Foundation of China under Grant No. 61202031, 60970017, 61100017, the National Science \& Technology Pillar Program of China under grant No.2012BAH07B01, the central grant funded Cloud Computing demonstration project of China undertaken by Kingdee Software (China).

\section{References}

1. Eriksson, H.-E., Penker, M.: Business Modeling with UML: Business Patterns at Work, John Wiley \& Sons, Inc (2000)

2. Fukazawa, Y., Naganuma, T., Kurakake, S.: Construction and Use of Role-ontology for Task Based Service Navigation System. In: Proc. of 2006 Internation Semantic Web Conference, pp. 806-819 (2006)

3. Ghose, A., Koliadis, G.: Actor eco-systems: From high-level agent models to executable processes via semantic annotations. In: Proc. of IEEE International Computer Software and Applications Conference 2007, pp. 177-184 (2007)

4. Grund, M., Schapranow, M., Krueger, J. et al.: Shared Table Access Pattern Analysis for Multi-Tenant Applications. In: Proc. of 2008 Advanced Management of Information for Globalized Enterprises, pp.1-5 (2008) 
5. He, K., Wang, J., Liang, P.: Towards Semantic Interoperability Aggregation in Service Requirements Refinement. Journal of Computer Science and Technology, 25(6), 1103-1117 (2010)

6. Jureta, I., Faulkner S.: An agent-oriented meta-model for enterprise modeling. In: Proc. of ER workshop 2005, LNCS 3770, pp.151-161, Springer, Heidelberg (2005)

7. Kang, K., Cohen, S., Hess, J., et al.: Feature-Oriented Domain Analysis (FODA): feasibility study. Technical Report: CMU/SEI-90-TR-021, Software Engineering Institute/Carnegie Mellon University(1990)

8. Klusch, M., Kaufer, F.: WSMO-MX: A Hybrid Semantic Web Service Matchmaker. Web Intelligence and Agent Systems, 7(1), 23-42 (2009)

9. Koliadis, G., Ghose, A.: Relating business process models to goal-oriented requirements models in KAOS. In: Advances in Knowledge Acquisition and Management, vol. 4303, pp. 25-39. Springer, Heidelberg (2006)

10. Lamsweerde, A. V.: Goal-oriented requirements engineering: a guided tour. In: Proc. of the 5th IEEE Int. Symp. on Requirements Engineering (ER'01), pp. 249-263, Toronto, Canada (2001)

11. Lazovik, A., Ludwig, H.: Managing process customizability and customization: Model, language and process. In: Proc. of the 8th International Conference on Web Information Systems Engineering (WISE), Springer, Heidelberg (2007)

12. Liu, W., He, K., Wang, J., et al.: Heavyweight semantic inducement for requirement elicitation and analysis. In: Proc. of the 3rd International Conference on Semantics, Knowledge and Grid, pp. 206-211, Xi'an, China (2007)

13. Martin, D., Ankolekar, A., Burstein, M., et al. OWL-S: Semantic Markup for Web Services. W3C Candidate Recommendation, (2006) http://www.daml.org/services/owl-s/

14. Masolo, C., Vieu, L., Bottazzi, E., et al.: Social roles and their descriptions. In: Proc. of the 9th Int. Conf. on the Principles of Knowledge Representation and Reasoning, pp. 267-277 (2004)

15. Mell, P., Grance, T.:The NIST Definition of Cloud Computing. National Institute of Science and Technology. http://csrc.nist.gov/publications/nistpubs/800-145/SP800-145.pdf

16. Mietzner, R., Leymann, F.: Generation of BPEL Customization Processes for SaaS Applications from Variability Descriptors. In: Proc. of 2008 IEEE International Conference on Services Computing (SCC), pp. 359-366. Hawaii, United States (2008)

17. Mili, H., Mili, F., Mili, A.: Reusing software: issues and research directions. IEEE Transactions on Software Engineering, vol. 21, 528-562 (1995)

18. OASIS.:Web Services Business Process Execution Language Version 2.0, OASIS Stan$\operatorname{dard}(2007)$ http://docs.oasis-open.org/wsbpel/2.0/

19. Roberto Chinnici,Jean-Jacques Moreau, Arthur Ryman, et al.:Web Services Description Language (WSDL) Version 2.0, W3C Recommendation. http://www.w3.org/TR/wsdl20/

20. Rodriguez, M.A., Egenhofer, M.J.: Determining semantic similarity among entity classes from different ontologies, IEEE Transactions on Knowledge and Data Engineering, 15(2), pp. 442-456(2003)

21. Van der Aalst, W. M. P., Dreiling, A., Gottschalk, F., et al.: Configurable process models as a basis for reference modeling. In: Proc. of Business Process Management Workshops, pp. 512-518. Springer, Heidelberg (2005)

22. Wang, J., He, K., Gong, P., et al.: RGPS: A Unified Requirements Meta-Modeling Frame for Net-worked Software. In: Proc. of the 3rd International Workshop on Advances and Applications of Problem Frames at 30th ICSE, pp. 29-35. Leipzig, Germany (2008)

23. Wang, J., Zhang, J., Hung, P.C.K., et al.: Leveraging Fragmental Semantic Data to Enhance Services Discovery. In: Proc. of the 13th IEEE International Conference on High Performance Computing and Communications, pp.687-694, Banff, Canada (2011)

24. Zhang, L.-J., Zhang, J., Cai, H.: Services Computing. Springer (2007) 